\title{
Sliding mode control applied to improve the dynamical response of a spherical 3D wind sensor for Mars atmosphere
}

\author{
Maria-Teresa Atienza*, Lukasz Kowalski, Sergi Gorreta, Vicente Jiménez, Luis M. Castañer, Manuel Domínguez-Pumar \\ Micro and Nano Technologies Group, Electronic Engineering Department. Universitat Politècnica de Catalunya, Jordi Girona 1-3, 08034 Barcelona, Spain
}

\begin{abstract}
The objective of this paper is to show how the structure of a thermal wind anemometer can be tuned to ensure a fast response when the sensor works in closed loop configuration (constant temperature operation). If the thermal filter associated to the sensor structure has only one significative time constant, the resulting system time response, working in closed loop, is enhanced beyond the natural limit imposed by its own thermal circuit. This effect is theoretically explained using the theory of sliding mode controllers. Experimental corroboration is presented by comparing the results obtained with two prototypes of a spherical wind sensor for Mars atmosphere. It will be shown that in case of having only one significant time constant, the time response in closed loop is much faster than the value associated with that time constant. It will be experimentally shown that this effect is lost when the thermal filter has more than one significant time constant. Diffusive representation is used to obtain arbitrary-order models of the thermal structures of the sensors.
\end{abstract}

Key words: Wind sensors, sliding mode controllers, diffusive representation, time-varying systems

\section{Introduction}

The objective of this paper is to present the improvements of the dynamical response of a spherical 3D wind sensor for Mars atmosphere. Wind sensing in Mars is a challenging task due to the rarefied atmosphere, mostly composed of $\mathrm{CO}_{2}$, low pressure (in the range of 6-12mBar) and large temperature dynamical range: from 150 to $300 \mathrm{~K}$ [1]. Several methods exist for ${ }_{15}$

\footnotetext{
${ }^{*}$ Corresponding author

Email addresses: maria.teresa.atienza@upc.edu (Maria-Teresa Atienza )
}

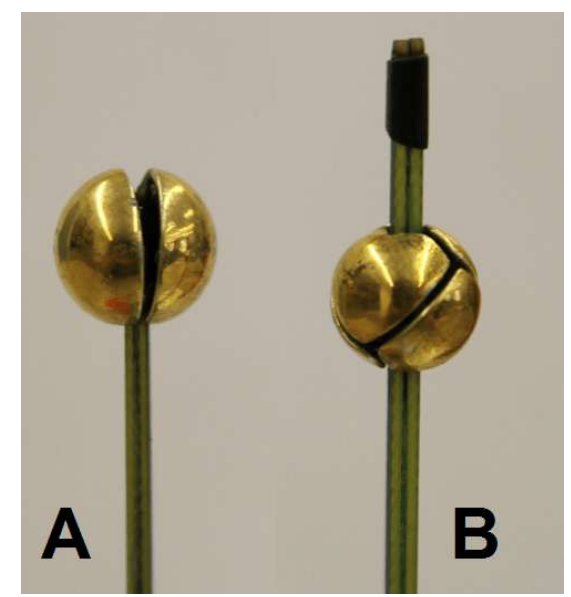

Figure 1: Left: Sensor A. Photo of previous two sector design wind sensor. Right: Sensor B. Photo of new four sector design wind sensor. measuring wind speed and direction on Mars surface. Thermal anemometry, though, is for now the most adequate method in this environment. Sensors based on ultrasound do not work well below $15 \mathrm{mBar}$ and require higher powers to operate [2]. Windsocks [3] and tell-tales [4] have been also used for detecting wind velocity and angle, but these methods require to process images to infer wind patterns. Thermal anemometry, then benefits from a general simplicity and robustness. This method detects the wind velocity by measuring the power losses of a heated element due to forced convection. These thermal sensors were first introduced for Mars missions in Viking Lander [5, 6], in 1976. The wind sensor was formed by two small cylinders covered by a thin film of platinum that formed a resistance, which worked under constant temperature mode. The Pathfinder mission of 1997 [7, 3] also included a wind detection sensor based on thermal anemometry. It consisted in a cylindrical mast with six platinum-iridium wires as heating elements. More recently, in August 2012, the Mars Science Laboratory (MLS) on board the Curiosity rover landed in the red planet. It included the REMS (Remote Environmental Monitoring Station) sensor suite that measures humidity, pressure, temperature, radiation and wind velocity $[8,9,10]$. For the wind velocity and direction detection, platinum resistors, fabricated on silicon technology, were employed. These resistors were grouped in sets of four in a coplanar plane, to achieve $2 \mathrm{D}$ wind sensitivity. The $3 \mathrm{D}$ direction was obtained placing three of these sets forming $120^{\circ}$ with each other in a cylindrical boom. Two of these booms were incoporated in REMS. The same device concept is scheduled to fly again in the InSight Mission in 2018, the TWINS instrument (Temperature and Wind sensors for InSight mission). While Viking was able to send data for two years, Pathfinder sent wind 
direction data only. REMS is still operational, although some of the chips were damaged when landing [10].

Two sensor prototypes of an spherical anemometer, heritage of the REMS wind sensor, will be described in this paper. The main advantages of these sensors are their spherical geometry, which eases the 3D wind speed recovery reverse algorithm [11],

45 and the simplicity of the system itself, with only a few heating 85 elements. Furthermore, as the heating elements are inside a metallic shell, the sensor is more robust [11]. Figure 1 shows a picture of both sensors.

As it has been mentioned above, the dynamics of the two 50 sensor prototypes is analyzed in this paper. In the more recent prototype, (Figure 1]B) the thermal coupling between the heating elements and the sectors conforming the shell of the sensor has ${ }_{90}$ been improved using bare platinum chip resistors instead of encapsulated commercial resistances (as in Figure 11A).

The purpose of this paper is to experimentally assess how the dynamical response of the sensor, following a wind speed change, is significantly improved due to the better thermal coupling between the hot dice and the shell exposed to the wind. 95 Moreover, in this paper we seek to find a general quantitative 60 condition that thermal anemometers have to fulfill in order to have a closed loop response much faster than the open loop thermal time constants. This is the main reason why we compare sensors $\mathrm{A}$ and $\mathrm{B}$ having different thermal coupling to the ${ }_{100}$ ambient.

To do so we have used the tools of Sliding Mode Control (SMC) to the sigma-delta modulator we have used to close the loop of the two sensors. It is well known in SMC theory that any disturbance (e.g. wind speed changes in our case) can have ${ }_{105}$ matched and unmatched components and, if only matched com70 ponents are present, the time response in the equivalent control is much faster. This is demonstrated to happen in sensor B and not in sensor A, as described in Section 5 below.

The SMC parameters are found from open loop measure-110 ments at several wind velocities using modelling based on Diffusive Representation. These state-space models have proven to be very well suited for thermal applications [12]. The extraction method is different from the one we have used in [13]
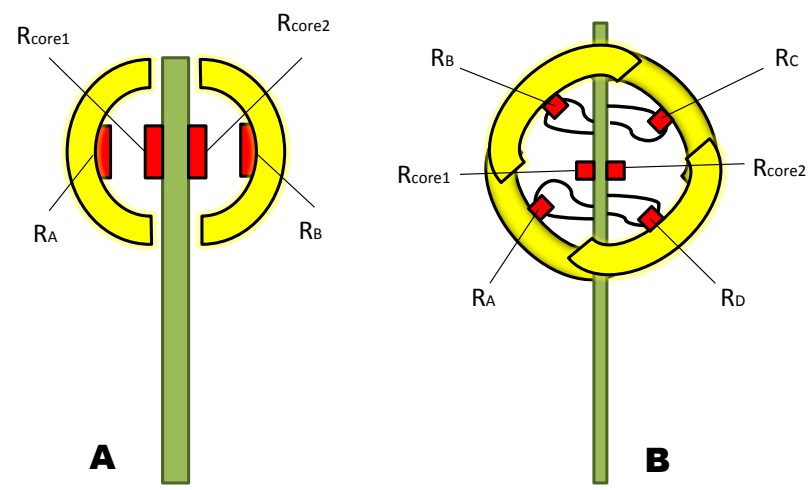

Figure 2: Left: Sensor A. Schema of first prototype of spherical wind sensor. Right: Sensor B. Schema of new desing of improved wind sensor in which each thermal dynamical model was obtained using an experiment at constant wind speed.

Both tools, SMC and DR, are used together and are explained in this paper. Section 2 describes the sensors and how they are operated. Section 3 presents the thermal models extracted from both sensors prototypes and the sliding mode analysis of how the system will respond under closed loop operation. Section 4 analyzes the conditions for obtaining a matched or a mismatched response in the sensor. Finally, experimental results are shown in section 5 .

\section{Sensor Description}

Figure 2 shows a schematic view of the two sensors. In the following, each sensor is going to be described briefly.

SENSOR A. The initially designed sensor is composed of two silver hemispheres connected to a PCB (Printed Circuit Board), which provides mechanical and electrical support. In order to reduce emisivity and avoid oxidation, the hemispheres are polished and sputtered with a thin layer of gold on their surface respectively. Each hemisphere integrates a SMD (Surface Mounted Device) Pt resistor. The resistors, $R_{A}$ and $R_{B}$, allow to heat the hemispheres and sense the temperature. In the PCB, two more Pt resistors are attached, $R_{\text {core }_{1}}$ and $R_{\text {core }_{2}}$, in order to heat the core of the sphere to reduce the conduction heat flux between the hemispheres and the supporting structure. The resistors of sensor A are commercial surface mounted $100 \Omega$ platinum resistors with temperature coefficient $\alpha=0.00385^{\circ} C^{-1}$ with different encapsulations; SMD0603 for the spherical sectors and SMD0805 for the PCB.

SENSOR $B$. The new design is composed of four silver sectors connected to a PCB. Now, the silver sectors (which have the same polishing and sputtering process as that of sensor A), form a tetrahedral sphere of same radius as the sphere formed in sensor A. Again, each sector and the PCB have a Pt resistor integrated on them $\left(R_{A}, R_{B}, R_{C}\right.$ and $R_{D}$ in the sectors, and $R_{\text {core }}$ and $R_{\text {core }_{2}}$ in the PCB), which allow heating and sensing of the temperature and reducing conduction heat flux. A key design factor for improving the time response of the sensor is to ensure a good thermal connection between heaters and sectors. To achieve this goal non-encapsulated chip resistors have been used this time. Additionaly, the electrical conexion between the PCB and the resistors is made by a wire bonding process. For this 20 purpose, resistors have been fabricated in our clean room using a Silicon wafer. Silicon oxide $\left(\mathrm{SiO}_{2}\right)$ has been grown thermally for electrical isolation. On top of the $\mathrm{SiO}_{2}$, the platinum resistors were patterned with a photolitography process. The Pt resistors of sensor B have a nominal value of $175 \Omega$ and a temperature coefficient $\alpha=0.0031^{\circ} C^{-1}$.

\subsection{Sensor Operation}

Thermal anemometers can work in open or closed loop operation mode. In open loop mode, a constant current or voltage is injected into the heaters of the sensor while the temperature 
due to convection losses to the fluid, are measured to infere the wind speed. In the closed loop, however, the temperature in the heated elements is forced to a constant value (CTA mode, Constant Temperature Anemometry), and the power required at every sector to keep constant the temperature is the output signal ${ }_{140}$ of the sensor. The wind velocity is calculated from the thermal conductance (ratio of the power and the temperature difference between the hot point and the ambient) which is related to the Nusselt number (embodying the Reynolds number and hence the wind speed). It is known that constant temperature operation mode is better in terms of time response [8]. In section 5 of this paper, both operation modes are tested and compared.

$$
\mathbf{A}=\left[\begin{array}{cccccccc}
\sum_{n: g(n)=w_{1}} \psi_{1}^{(n)}\left(t_{0}\right) & \ldots & \sum_{n: g(n)=w_{1}} \psi_{K}^{(n)}\left(t_{0}\right), & \ldots & \sum_{n: g(n)=w_{J}} \psi_{1}^{(n)}\left(t_{0}\right) & \ldots & \sum_{n: g(n)=w_{J}} \psi_{K}^{(n)}\left(t_{0}\right), & {[1]_{k}} \\
\vdots & \ddots & \vdots & \vdots & \vdots & \ddots & \vdots & \vdots \\
\sum_{n: g(n)=w_{1}} \psi_{1}^{(n)}\left(t_{F}\right) & \cdots & \sum_{n: g(n)=w_{1}} \psi_{K}^{(n)}\left(t_{F}\right), & \cdots & \left.\sum_{n: g(n)=w_{J}} \psi_{1}^{(n)}\left(t_{F}\right)\right] & \cdots & \sum_{n: g(n)=w_{J}} \psi_{K}^{(n)}\left(t_{F}\right), & \left.\left[e^{-\xi_{k}\left(t_{F}-t_{0}\right)}\right]_{k}\right]
\end{array}\right]
$$

\section{Dynamical Analysis}

An analysis of the dynamics of the sensor structures has been carried out to understand and predict the time response of each sensor. In this section we present the tools of diffusive representation and sliding mode controllers, that will be used together. The same modelling and analysis method has been employed both in sensors A and B.

\subsection{Thermal modelling: Diffusive Representation}

The theory of Diffusive Representation allows obtaining exact and approximate state realizations of a wide class of integral operators. This mathematical tool is suitable for system identification of any physical phenomena based on diffusion [14].

Given a non-rational transfer function, $H(p)$, associated with a convolution causal operator denoted by $H\left(\partial_{t}\right)$, the diffusive realization of this operator is expressed by the following input $(u)$ - output (y) state space realization of $u \longmapsto y=H\left(\partial_{t}\right) u=h * u$ of the form [15]:

$$
\begin{aligned}
\frac{\partial \psi(\xi, t)}{\partial t} & =-\xi \psi(\xi, t)+u(t), \quad \psi(\xi, 0)=0 \\
y(t) & =\int_{0}^{\infty} \eta(\xi, t) \psi(\xi, t) d \xi
\end{aligned}
$$

where $\xi \in \mathbb{R}$ is frequency, $\eta(\xi, t)$ is the diffusive symbol of $H\left(\partial_{t}\right)$ that represents how the system behaves, and the statevariable $\psi(\xi, t)$ is a time-frequency representation of the input, called the diffusive representation of $u(t)$ [15].

To be able to handle experimental data, a discrete approximation of $H\left(\partial_{t}\right)$ can be built discretizing the continuous variable $\xi$ into $\left\{\xi_{k}\right\}_{1 \leq k \leq K}$, where $K$ is the order of the discretized model. This leads to an input - output approximation $u \longmapsto \tilde{y} \approx y=$ $H\left(\frac{d}{d t}\right) u$. The dynamical system can be described by:

$$
\begin{array}{lll}
\psi_{k}^{(n)}(t)=0 & t \in\left[t_{0}, t_{n}\right] \\
\dot{\psi}_{k}^{(n)}(t)=-\xi_{k} \psi_{k}^{(n)}+u(t) & t \in\left[t_{n}, t_{n+1}\right] \\
\dot{\psi}_{k}^{(n)}(t)=-\xi_{k} \psi_{k}^{(n)} & t \geq t_{n+1} \\
\tilde{y}(t)=\sum_{n, k} \eta_{k}^{(n)} \psi_{k}^{(n)}(t)+\sum_{k}^{K} c_{k} e^{-\xi_{k}\left(t-t_{0}\right)}
\end{array}
$$

where $\eta_{k}^{(n)} \in \mathbb{R}^{K}$ is the diffusive symbol associated to the conditions of the system in the $n$-th interval in $t \in\left[t_{n}, t_{n+1}\right] . c_{k} \in \mathbb{R}^{K}$ represents the initial conditions of the system at the beginning of the measurements, at $t=t_{0}$. These intervals are a discretization of time for which the dissusive symbols can be considered contant in a wind experiment. This model extraction method, is different from the method described in [13], where the diffusive symbols for each wind velocity were inferred from long openloop measurements at a single wind velocity. In that case, every time the wind was switched, the memory of the system was lost, and therefore, it was necessary to find new initial conditions. It was not possible to predict the thermal dynamics without having before hand the closed-loop measurements under switching wind.

On the contrary, in this paper, the thermal characterization of the wind sensors is made from open-loop measurements in which wind speed is being continuously switched between several wind velocities. The diffusive symbols corresponding to each wind velocity are hence inferred from a single experiment, with $t \in\left[t_{0}, t_{F}\right]$. The number of wind velocities for which the sensor will be characterized is $J$. The wind speeds, $\left\{w_{1}, \ldots, w_{J}\right\}$, are a uniformly distributed random sequence. Each wind speed is applied in an interval $t \in\left[t_{n}, t_{n+1}\right]_{n=0, \ldots, N}$, where $N$ is the number of wind events, such that, $N \gg J$. All the wind velocities are applied for a short time $\Delta t_{n}=\left(t_{n+1}-t_{n}\right)$, where $\Delta t_{n} \ll t_{F}$, for all $n$. In the meantime, a PRBS heating current is applied to the resistors in open-loop, with a period $T_{P R B S} \ll \Delta t_{n} \ll t_{F}$.

The solution to the identification problem is found solving the finite least squares problem:

$$
\min _{\eta \in \mathbb{R}^{K}}\|\mathbf{A} \hat{\boldsymbol{\eta}}-\mathbf{Y}\|^{2}
$$

where $\mathbf{A}$ is of the form of Eq. 11 and the vector $\hat{\boldsymbol{\eta}}^{T}$ is that of Eq. (5).

In matrix of Eq. (1), the function $g(n)$ returns the wind $w_{j}$ applied at every wind interval $n$. The last column of this matrix, $[1]_{k} \in \mathbb{R}^{K}$ is an all ones vector and

$$
\left[e^{-\xi_{k}\left(t-t_{0}\right)}\right]_{k}=\left[e^{-\xi_{1}\left(t-t_{0}\right)}, \ldots, e^{-\xi_{K}\left(t-t_{0}\right)}\right]
$$

The diffusive symbol vector that is going to be inferred is of the form of Eq. (5):

$$
\hat{\boldsymbol{\eta}}^{T}=\left[\left[\eta_{1}^{w_{1}}, \ldots, \eta_{K}^{w_{1}}\right]^{T}, \ldots,\left[\eta_{1}^{w_{J}}, \ldots, \eta_{K}^{w_{J}}\right]^{T},\left[c_{1}, \ldots, c_{K}\right]^{T}\right]
$$

where $\left[\eta_{k}^{w_{j}}\right]^{T}$ is the diffusive symbol corresponding to the wind $w=w_{j}$. The measurements vector is $\mathbf{Y}^{T}=\left[\begin{array}{lll}T\left(t_{0}\right), & \ldots, & T\left(t_{F}\right)\end{array}\right]$, 
being $T\left(t_{i}\right)$ the temperature in the instant $t_{i} \in\left[t_{0}, t_{F}\right]$ in our case. The solution to the inference problem is classically given by: ${ }_{240}$

$$
\hat{\boldsymbol{\eta}}=\left[\mathbf{A}^{*} \mathbf{A}\right]^{-1} \mathbf{A}^{*} \mathbf{Y}
$$

\subsection{Constant Temperature Anemometer dynamics}

The analysis of the heat flow dynamics in an anemometer is 245 contemplated under constant temperature mode operation. This study will be undertaken considering that the controller is a sigma-delta modulator, which is a well-known type of analog-todigital converter [16]. A thermal sigma-delta modulator, applies a modulated power to maintain the temperature constant in the or shell. In this control, at each sampling period, $T_{s}$, the temperature of the hot element, $T_{n}=T\left(n T_{s}\right)$, is compared to the desired target temperature, $\Delta T$. If $T_{n} \geq \Delta T, P_{\text {off }}$ is delivered ${ }^{250}$ during the following sampling period, and if $T_{n}<\Delta T, P_{\text {on }}$ is injected instead. Sigma-delta modulators can be seen as an ple of a sliding mode controller (SMC), 117]. These type of controllers change the dynamics by applying a discontinuous control signal so that, under some conditions, the system "slides" on a certain control surface [13]. In the case of sigma-delta modulation, the typical hysteresis used in SMC is substituted by a discretization of time.

To analyze the dynamics of the system for the general case of finite order thermal systems a control surface has to be defined. This is:

$$
\sigma(\psi(t))=\Delta T-\tilde{y}(t)=\Delta T-\sum_{k}^{K} \eta_{k}(t) \psi_{k}(t)
$$

The objective is to place the system within the control surface $\sigma(\psi(t))=0$ in finite time, in our case to have a constant temperature. This is possible if, [13]:

$$
P_{\text {off }} \sum_{k}^{K} \frac{\eta_{k}(t)}{\xi_{k}}<\Delta T<P_{\text {on }} \sum_{k}^{K} \frac{\eta_{k}(t)}{\xi_{k}}
$$

The condition $\dot{\sigma}(\psi(t))=0$ gives the necessary equivalent control under a sliding motion to maintain the temperature of the shell constant and for a system described by diffusive representation is given by Eq. (9):

$$
u_{e q}(t)=\Gamma^{-1} \sum_{k}^{K} \eta_{k}(t) \xi_{k} \psi_{k}(t)
$$

where $\Gamma=\sum_{k}^{K} \eta_{k}(t)$.

Then, the dynamics of the system in the control surface is determined when the equivalent control is applied:

$$
\dot{\psi}_{k}(t)+\xi_{k} \psi_{k}(t)=\Gamma^{-1} \sum_{l}^{K} \eta_{l}(t) \xi_{l} \psi_{l}(t)
$$

If the equilibrium point $\left(\dot{\psi}_{k}(t)=0\right)$ is reached, the system takes the steady-state values $\psi_{Q}\left(\xi_{k}\right)=\frac{u_{Q}}{\xi_{k}}$ and $u_{Q}=\frac{\Delta T}{\sum_{k}^{K} \eta_{k} / \xi_{k}}$.

The time evolution predicted by the SMC analysis is obtained taking into account the changes in wind speed. The equivalent control to maintain the desired target temperature can be 27 predicted using the diffusive symbols inferred in the open-loop characterization. The wind velocities for which the analysis will be done must be the same as the ones for which the wind sensor has been characterized previously. Now, in this experiment, the wind is kept constant within the time intervals $\Delta t_{n}^{\prime}=\left(t_{n+1}^{\prime}-t_{n}^{\prime}\right)$, for $n=0, \ldots N^{\prime}$, where $N^{\prime}$ is the number of wind intervals such as $N^{\prime} \geq J$.

The first step to calculate the equivalent control is to obtain the point $t_{c}^{\prime} \in\left(t_{0}^{\prime}, t_{1}^{\prime}\right)$ at which the system reaches the control surface, $\sigma=0$. From a zero initial condition, a constant $u\left(t^{\prime}\right)=P_{\text {on }}$ is applied till the control surface is reached. The intersection point of this initial trajectory with the surface is the initial condition for the sliding movement on the control surface following expressions (9) and (10) for the first wind step of the experiment. Now, Eq. (10) can be expressed as:

$$
\dot{\psi}_{k}\left(t^{\prime}\right)=-D\left(t^{\prime}\right) \psi\left(t^{\prime}\right), \quad t^{\prime} \geq t_{c}^{\prime}
$$

where $D\left(t^{\prime}\right)$ collects the terms in Eq. (10). Since wind velocities are considered constant during each interval $\Delta t_{n}^{\prime}$ of the experiment, $D\left(t^{\prime}\right)$ is piecewise constant and therefore for $t^{\prime} \in\left[t_{n}^{\prime}, t_{n+1}^{\prime}\right]$

$$
\psi\left(t^{\prime}\right)=e^{-D^{(n)}\left(t^{\prime}-t_{n}^{\prime}\right)} \psi\left(t_{n}^{\prime}\right)
$$

where:

$$
D^{(n)}=\Gamma^{-1}\left[\begin{array}{ccc}
\eta_{1}^{(n)} \xi_{1}-\Gamma \xi_{1} & \ldots & \eta_{K}^{(n)} \xi_{K} \\
\vdots & \ddots & \vdots \\
\eta_{1}^{(n)} \xi_{1} & \ldots & \eta_{K}^{(n)} \xi_{K}-\Gamma \xi_{K}
\end{array}\right]
$$

with $\eta_{k}^{(n)}$ the diffusive symbol of the sensor working under the wind of the $n$-th time interval.

Replacing Eq. (12) in the equivalent control expression of Eq. (9) we reach:

$$
u_{e q}\left(t^{\prime}\right)=\mathbf{H}\left(t^{\prime}\right) \psi\left(t_{c}^{\prime}\right)
$$

where:

$$
\mathbf{H}\left(t_{n}^{\prime}\right)=\Gamma^{-1} \sum_{k}^{K} \eta_{k}\left(t^{\prime}\right) \xi_{k} e^{-D^{(n)}\left(t_{n}^{\prime}-t_{n-1}^{\prime}\right)} e^{-D^{(n-1)}\left(t_{n-1}^{\prime}-t_{n-2}^{\prime}\right)} \cdots e^{D^{(1)}\left(t_{1}^{\prime}-t_{c}^{\prime}\right)}
$$

where it has been assumed that $t_{1}^{\prime}>t_{c}^{\prime}$.

The extracted diffusive symbols from 3.1 allow to predict the system behaviour under closed-loop operation control in the presence of wind variations. The equivalent control to maintain constant the temperature is directly obtained from the application of Eq. (13).

\section{Reduction of the number of state variables for ensuring matched disturbances for time response acceleration}

Wind velocity changes can be seen as an external disturbancee of the system, represented by an additional tem: $\theta_{k}(\psi, t)=$ $\left(\theta_{1}(\psi, t), \cdots, \theta_{N}(\psi, t)\right)^{T}$. Since, $\psi_{k}(0)=0$, the thermal system can also be expressed as [13]:

$$
\begin{aligned}
\dot{\psi}_{k}(t)= & -\xi_{k} \psi_{k}(t)+\frac{\eta_{k}}{2}\left(P_{\mathrm{off}}+P_{\mathrm{on}}\right) \\
& +\frac{\eta_{k}}{2}\left(P_{\mathrm{on}}-P_{\mathrm{off}}\right) u+\theta_{k}(\psi, t) \\
\sigma(\psi(t))= & \Delta T-\sum_{k}^{N} \psi_{k}(t)
\end{aligned}
$$



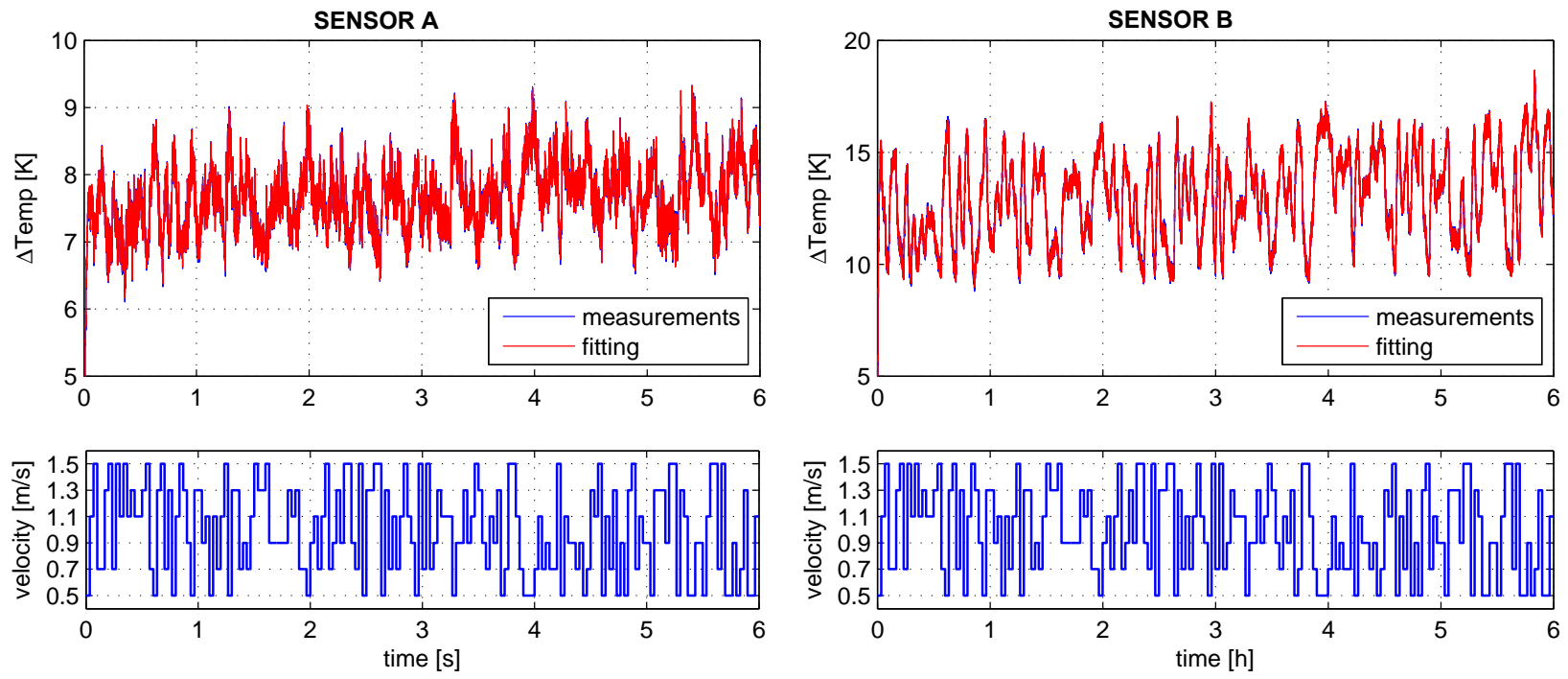

Figure 3: Sensor A. Top: Experimental data Vs Fitting data along the six hours of the experiment in open-loop mode (they are almost undistinguishable). Bottom: Wind velocity as a function of time. Sensor B. Top: Experimental data Vs Fitting data along the six hours of the experiment in open-loop mode (they are almost undistinguishable). Bottom: Wind velocity as a function of time.
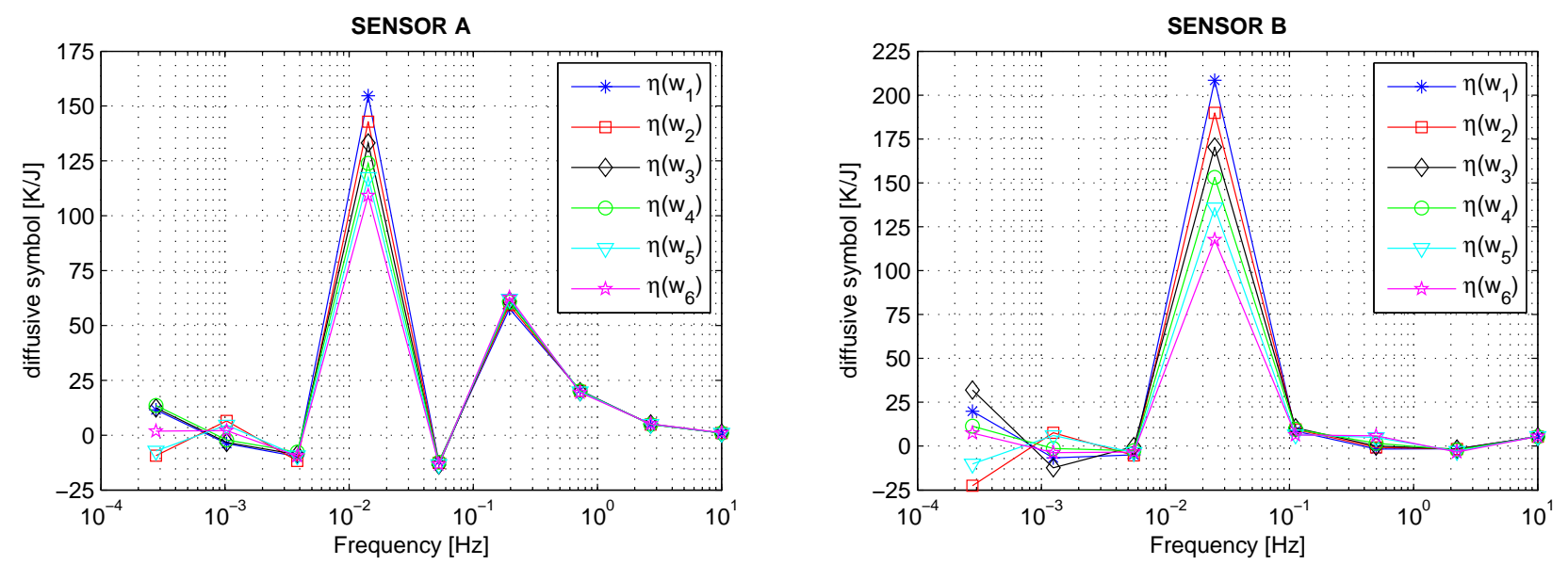

Figure 4: Left: Sensor A. 9-order diffusive symbols for each wind velocity of the experiment. Right: Sensor B. 8-order diffusive symbols for each wind velocity of the experiment. $w_{1}=0.5 \mathrm{~m} / \mathrm{s}, w_{2}=0.7 \mathrm{~m} / \mathrm{s}, w_{3}=0.9 \mathrm{~m} / \mathrm{s}, w_{4}=1.1 \mathrm{~m} / \mathrm{s}, w_{5}=1.3 \mathrm{~m} / \mathrm{s}$ and $w_{6}=1.5 \mathrm{~m} / \mathrm{s}$.

where $u=\operatorname{sgn}\left(\sigma^{\prime}(\psi(t))\right.$ and the control surface is $\sigma^{\prime}(\psi(t))=$ $\Delta T-\sum_{k} \psi_{k}(t)$.

Assuming the sliding motion is taking place, this is $d \sigma^{\prime} / d t=$ 0 , the equivalent control is now:

$$
u_{\mathrm{eq}}=\frac{\sum_{k}^{N} \xi_{k} \psi_{k}(t)-\frac{\Gamma}{2}\left(P_{\mathrm{off}}+P_{\mathrm{on}}\right)-\chi(\psi, t)}{\frac{\Gamma}{2}\left(P_{\mathrm{on}}-P_{\mathrm{off}}\right)} \in[-1,1]
$$

with $\chi(\psi, t)=\sum_{k} \theta_{k}(\psi, t)$. The new dynamics in the control surface is therefore determined by:

$$
\begin{aligned}
\dot{\psi}_{k}(t)= & -\xi_{k} \psi_{k}(t)+\frac{\eta_{k}}{2}\left(P_{\mathrm{off}}+P_{\mathrm{on}}\right)+\theta_{k}(\psi, t) \\
& +\frac{\eta_{k}}{\Gamma}\left[\sum_{l} \xi_{l} \psi_{l}(t)-\frac{\Gamma}{2}\left(P_{\mathrm{on}}+P_{\mathrm{off}}\right)-\chi(\Psi, t)\right]
\end{aligned}
$$

As it is well known, disturbances can be decomposed in a matched and a mismatched component [17]. In the case of a matched disturbance, $\theta_{k}(t)-\frac{\eta_{k}}{\Gamma} \chi(\psi, t)=0$, the disturbance is proportional to the diffusive symbol, i.e. $\theta_{k}(\psi, t)=\alpha \eta_{k}$ for some $\alpha \in \mathbb{R}$. Under this condition, the control method will be able to handle successfully the disturbances with a fast (almost instantaneous) response, since the dynamics of the system on the control surface would remain undisturbed. However, in the general case where a mismatched component is present, any disturbance will generate a change in the dynamics of the system on the control surface, therefore generating a slow dynamics in the equivalent control. Usually, the dynamics triggered by a mismatched component is expected to be of the same order as the one in the open loop response, i.e., slow.

One way of ensuring that any disturbance is a matched disturbance is the very simple case of having one state variable. This means that in a general case, it is very convenient to have only 


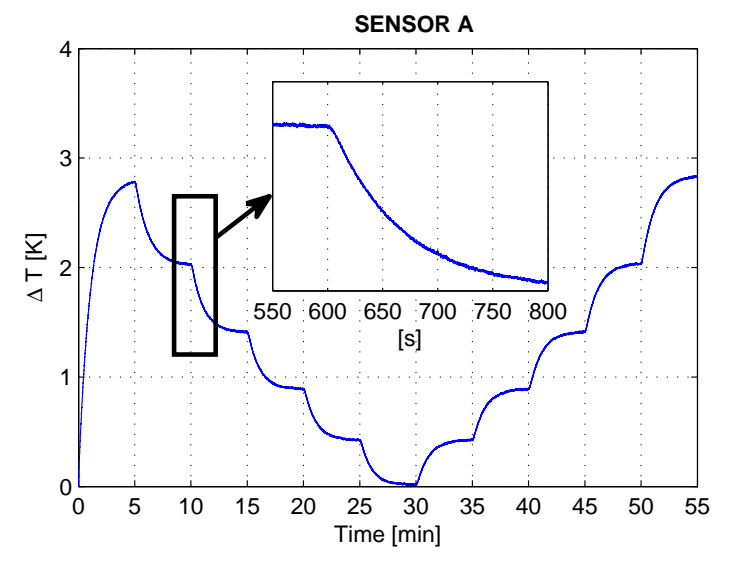

Figure 5: Temperature variation in one of the sectors of sensor A when the wind changes its velocity and the injected current is constant

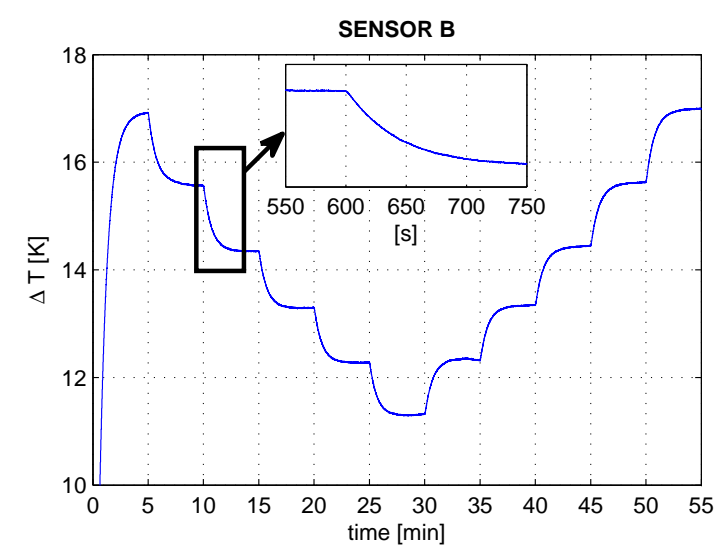

Figure 6: Temperature variation in one of the sectors of sensor B when the wind changes its velocity and the injected current is constant

one significant state variable of the thermal system, or what is is $^{345}$ the same, only one node of temperature in the interface between the heaters and the sensor's sectors. This is achieved in our prototypes if the thermal connection between the heated devices and the sectors is good enough. Experimental corroboration of this fact is shown in next section, with the thermal models of the sensors obtained using Diffusive Representation.

\section{Experimental Results}

Open and closed-loop experiments have been carried out to compare the time response of both sensors. For the experiments, the sensors were enclosed separately in an hypobaric stainless steel chamber. The pressure of the chamber was set to $45 \mathrm{mBar}$ and the measurements were made at room temperature. An auto-360 matic fan, located in front of the sensor, provides the necessary wind changes.

\subsection{Thermal model extraction} wind velocity at each sensor are shown in Figure 4 . As it can be
obsive observed, the diffusive symbol of sensor A has two significant maxima at approximately $f \approx 0.015 \mathrm{~Hz}$ and $f \approx 0.2 \mathrm{~Hz}$. In sensor $\mathrm{B}$, however, the maximum appears only at $f \approx 0.025$ Hz. The fact that the diffusive symbol of sensor B has only one significant pole, imply that the disturbances generated by the wind are matched, since the condition $\theta_{k}(\psi, t)=\alpha \eta_{k}$ is accomplished because there is only one significant variable. This means that the thermal system has a single node of temperature in the interface between the heating devices and the tetrahedral sectors. As it will be seen below in another experiment, the reduction to only one significant time constant will lead to a faster time response of this sensor under closed loop, where the 55 control will almost instantaneously absorb the changes due to wind variations.

Additionally, both sensors have been tested in open loop mode. In Figures 5 and 6 , the temperature changes corresponding to wind velocity changes when a constant current is being injected in the hemispherical and the tetrahedral sensor respectively can be observed. A $15 \mathrm{~mA}$ current has been injected into a sector of both sensors while the rest of the resistances were controlled to a constant temperature. The changes in the wind velocities went from 0.5 to $1.5 \mathrm{~m} / \mathrm{s}$ in ascendant steps of $0.2 \mathrm{~m} / \mathrm{s}$ 
$300 \mathrm{~s}$ and equally from 1.5 to $0.5 \mathrm{~m} / \mathrm{s}$ in descending order. As it can be seen in the inner figures, the open loop time response is slow, approximately 150 s for sensor A and 100s for sensor B. In open-loop configuration the time response difference between both sensors is not substantial, and the one that exists is probablyз9 than the sectors in A they will have less mass and will heat up faster.
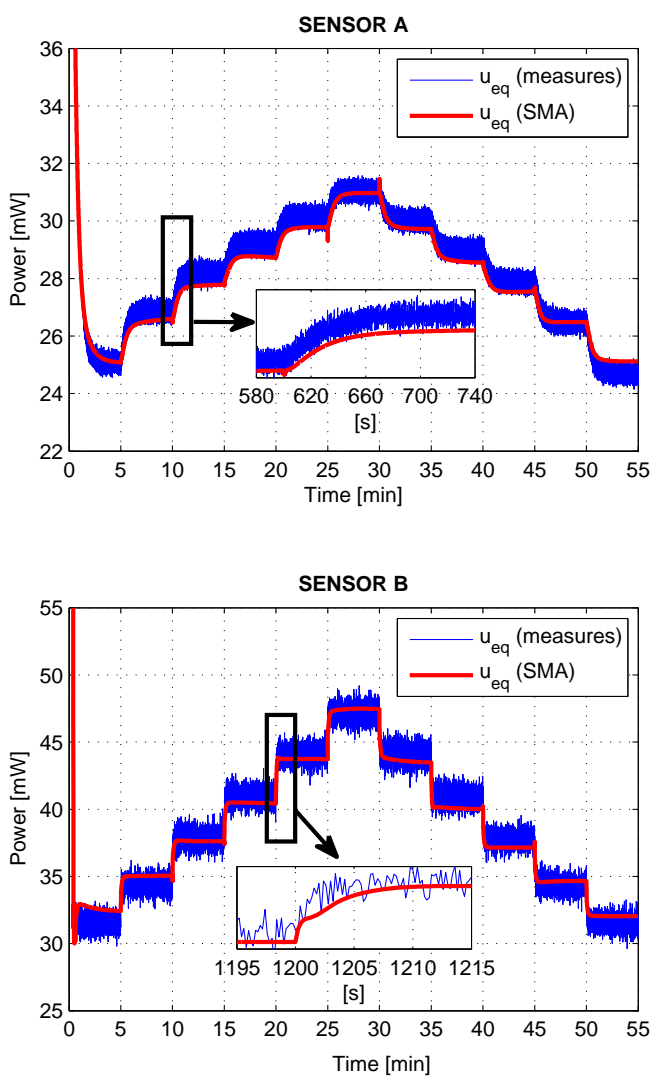

Figure 7: Top: The exprimental average power injected into the heater of sensor $\mathrm{A}$ in blue and the the result of applying the sliding mode analysis using the diffusive symbols of the left of Fig. 4 in red. Time response to a wind change is approximately 100s. Bottom: The experimental average power injected into the heater of sensor B in blue, and the result of applying the sliding mode analysis ${ }^{420}$ using the diffusive symbols of the right of Fig. 4 in red. Time response to a wind change is approximately $5 \mathrm{~s}$.

\subsection{Analysis of closed loop operation}

Under closed-loop operation mode, the system is maintaned at a constant target temperature $\Delta T=7.2 \mathrm{~K}$ for sensor $\mathrm{A}$ and $\Delta T=11.5 \mathrm{~K}$ for sensor $\mathrm{B}$. The wind velocities, the sames for which the system has been already characterized, changed every 430 300s with the same wind steps as in the constant current openloop experiment. In Figure 7 the experimental control waveform for sensor A (top) and B (bottom) is shown together with the sliding mode analysis prediction. As it can be observed there is a good match between the predictions of the SMC theory using ${ }_{435}$ the thermal models based on Diffusive Representation.

On the other hand. the time response to a wind change is of approximately $90 \mathrm{~s}$ in sensor A and of $5 \mathrm{~s}$ in case of sensor B (note that the fan has some few seconds of delay until the wind speed is achieved). Note that the time response in closed loop $(5 \mathrm{~s})$ is much smaller than the most significant time constant of sensor B (100s). This experimental result indeed confirms the predictions made in the previous sections. Sensor A, presents more than one time constant, and therefore the main gain of using a closed loop operation is not obtained since this implies the presence of mismatched components in the disturbances generated by the wind.

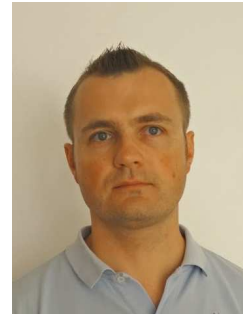

Maria-Teresa Atienza received the B.Sc. degree in Electronic Engineering and the M. Sc. degree in Physics Engineering from the Basque Country University, Spain, in 2012 and 2013 respectively. She joined Micro and Nano Technologies Research Group of the Universitat Politécnica de Catalunya (UPC), Spain, as a Ph.D. degree student where she is working on the identification of thermal systems applied to wind sensors for space applications.

Lukasz Kowalski obtained MSc degree in Electronics and Telecommunications from the Technical University of Lodz, Poland in 2005. He obtained $\mathrm{PhD}$ from Universitat Politécnica de Catalunya, Spain in 2016 for thesis 'Contribution to advanced hot wire wind sensing'. He has joined MNT group in September 2005. He has participated in space exploration projects: REMS(MSL), MEIGA (MetNet), MEDA (Mars2020). His work is aimed to the development of thermal anemometers for the atmosphere of Mars. His research areas include dimensionless analysis, FEM/CFD simulations, thermal modeling, inverse algorithm optimization for wind speed and incidence angle retrieval. $\mathrm{He}$ has co-authored two patents and several scientific papers. 


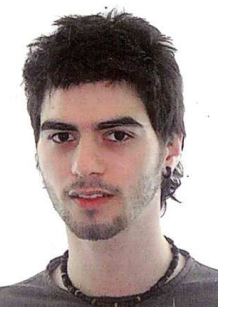

Sergi Gorreta received the M.Sc. degree in Telecomunication Engineering and the M.Sc. degree in Electronic Engineering from the Universitat Politècnica de Catalunya (UPC), Spain, in 2010 and $_{500}$ 2011 respectively. He is currently with the Micro and Nano Technologies Research Group of the UPC as a Ph.D degree student. His working areas are sensors for space applications, integrated circuit design, non linear circuits505 for MEMS and control of dielectric charging in MEMS switches.

450

Vicente Jiménez received the M.Sc. degree in 1992 and the $\mathrm{Ph}$, degree in 1997 from the Universitat Politècnica de d10 Catalunya (UPC), Barcelona, Spain. He has been with the Electronic Engineering Department, UPC, since 1992, when he became Associate Professor. His research areas include digital BiCMOS de-515 sign, development of microsystem circuit

460 interfaces, and microsystem modelling. He has participated in industry and space projects related to liquid and gas thermal flowmeters. He has coauthored more than 20 scientific papers in international journals and conferences.

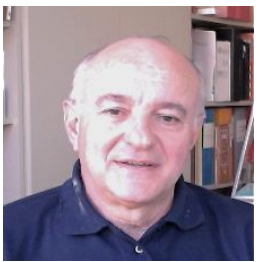

Luis M. Castañer is a Professor with the Departamento de Ingeniería Electrónica de la Escuela Técnica Superior de Ingenieros de Telecomunicación at the Universidad Politécnica de Cataluña, where he received his Doctor Ingeniero de Telecomunicación, following his undergraduate studies (Ingeniero Superior de Telecomunicación) from the Universidad Politécnica de Madrid (1971) and Diplome d'Etudes Approfondies (D.E.A.) en Physique Spatiale and Docteur-Ingenieur by the Université Paul Sabatier in Toulouse (1973). He has also been Dean of E.T.S.I.Telecomunicación and Head of Departamento de Ingeniería Electrónica, and has held several positions in Research Agencies and Committees: representative in the Comite de Gestion y Coordinacion of non nuclear energies, DGXII Comission of the EU, Head of the Programa Nacional de Tecnologías de la Información y Comunicaciones of the CICYT (1992- 1994), and Coordinator of the Technology Foresight at the Agencia Nacional de Evaluación y Prospectiva. He has contributed to semiconductor device research, covering solar cells in various aspects: technology of CIS, space degradation of Silicon and GaAs devices, and has contributed to the theory and technology of advanced bipolar transistors with polysilicon emitters and its application to high efficiency silicon solar cells, in particular ${ }_{550}$ to the emitter resistance of these devices. He has also contributed to the design, simulation and monitoring of photovoltaic power plants and systems, and is active in the microsystems technology area, working on flow sensors, dielectric degradation of $\mathrm{MEMS}_{555}$ electrostatic actuators and wind sensors for Mars. Recently he showing 3D self-assembly of nanoparticles assisted by electric field. He is a Senior IEEE Member, a Member of the Association and Charter of Telecommunication Engineers in Spain, and a Member of the Royal Spanish Engineering Academy.

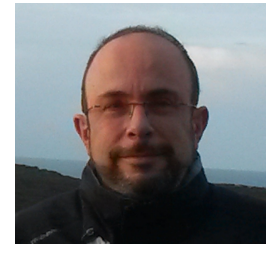

Manuel Domínguez-Pumar received the M.Sc. and Ph.D. degrees in electronic engineering from the Universitat Politécnica de Catalunya (UPC), Barcelona, Spain, and the M.Sc. (Hons.) degree in mathematics from the Universidad Nacional de Educación a Distancia, Madrid, Spain, in 1994, 1997, and 2005, respectively. Since 1994, he has been with the Department of Electronic Engineering, UPC, where he is currently an Associate Professor. From September 2006 to August 2007, he was a Visiting Scholar at the Courant Institute of Mathematical Sciences, New York, NY, USA.He is participating in the design of the MEDA wind sensor for NASA Rover2020. His research interests include control theory, chemical sensors, MEMS/NEMS sensors and actuators, dielectric charge control, sensors for space applications, sigma-delta modulation, and dynamical systems in general. Dr. Domínguez-Pumar received the 2013 NASA Group Achievement Award as a member of the Mars Science Laboratory REMS Instrument Development Team.

\section{References}

[1] L. Gottesdiener, Hot wire anemometry in rarefied gas flow Journal of Physics E: Scientific Instruments 13 (9) (1980) 908. URL http: //stacks.iop.org/0022-3735/13/i=9/a=001

[2] C. F. Wilson, Measurement of wind on the surface of mars, Ph.D. thesis, Linacre College, Oxford University (2003).

[3] R. Sullivan, R. Greeley, M. Kraft, G. Wilson, M. Golombek, K. Herkenhoff, J. Murphy, P. Smith, Results of the imager for mars pathfinder windsock experiment Journal of Geophysical Research: Planets 105 (E10) (2000) 24547-24562. doi:10.1029/1999JE001234 URL http://dx.doi.org/10.1029/1999JE001234

[4] H. P. Gunnlaugsson, C. Holstein-Rathlou, J. P. Merrison, S. Knak Jensen, C. F. Lange, S. E. Larsen, M. B. Madsen, P. Nørnberg, H. Bechtold, E. Hald, J. J. Iversen, P. Lange, F. Lykkegaard, F. Rander, M. Lemmon, N. Renno, P. Taylor, P. Smith, Telltale wind indicator for the mars phoenix lander Journal of Geophysical Research: Planets 113 (E3) (2008) n/a-n/a, e00A04. doi: 10.1029/2007JE003008 URL http://dx.doi.org/10.1029/2007JE003008

[5] T. E. Chamberlain, H. L. Cole, R. G. Dutton, G. C. Greene, J. E. Tillman, Atmopspheric measurements on mars: the viking meteorology experiment Bulletin of the American Meteorological Society 57 (9) (1976) 1094-1104. arXiv:http://dx.doi.org/ 10.1175/1520-0477(1976) 057<1094:AMOMTV>2.0.C0;2 doi:10 1175/1520-0477(1976) 057<1094: AMOMTV>2.0.CO;2 URL http://dx.doi.org/10.1175/1520-0477(1976) 057<1094: AMOMTV $>2.0 . \mathrm{CO} ; 2$

[6] R. Davey, T. Chamberlain, L. Harnett, Viking meteorology instrument sensor design analysis report, METC-021 Tech.

[7] M. P. Golombek, The mars pathfinder mission Journal of Geophysical Research: Planets 102 (E2) (1997) 3953-3965. doi : 10.1029/96JE02805 URL http://dx.doi.org/10.1029/96JE02805

[8] M. Domínguez, V. Jiménez, J. Ricart, L. Kowalski, J. Torres, S. Navarro, J. Romeral, L. Castañer, A hot film anemometer for the martian atmosphere Planetary and Space Science 56 (8) (2008) 1169 - 1179. doi:http://doi.org/10.1016/j.pss.2008.02.013 URL http://www.sciencedirect.com/science/article/pii/ S003206330800072X 
[9] J. Gómez-Elvira, C. Armiens, L. Castañer, M. Domínguez, M. Genzer, F. Gómez, R. Haberle, A.-M. Harri, V. Jiménez, H. Kahanpää, L. Kowalski, A. Lepinette, J. Martín, J. Martínez-Frías, I. McEwan, L. Mora, J. Moreno, S. Navarro, M. A. de Pablo, V. Peinado, A. Peña, J. Polkko, M. Ramos, N. O. Renno, J. Ricart, M. Richardson, J. Rodríguez-Manfredi, J. Romeral, E. Sebastián, J. Serrano, M. de la Torre Juárez, J. Torres, F. Torrero, R. Urquí, L. Vázquez, T. Velasco, J. Verdasca, M.-P. Zorzano,

565 J. Martín-Torres, Rems: The environmental sensor suite for the mars science laboratory rover Space Science Reviews 170 (1) (2012) 583-640. doi: 10.1007/s11214-012-9921-1

URL http://dx.doi.org/10.1007/s11214-012-9921-1

[10] S. Rafkin, J. Pla-Garcia, C. Newman, V. Hamilton, J. Martin-Torres, M. PazZorzano, H. Kahanpaa, E. Sebastian, The meteorology of gale crater determined from msl rems data and mesoscale modeling, in: Proc. 8th Int. Conf. Mars, 2014, pp. 1-2.

[11] L. Kowalski, M. T. Atienza, S. Gorreta, V. Jiménez, M. Domínguez-Pumar, S. Silvestre, L. M. Castañer, Spherical wind sensor for the atmosphere of 575 mars, IEEE Sensors Journal 16 (7) (2016) 1887-1897. doi:10.1109/ JSEN. 2015.2509168

[12] B. Allard, X. Jorda, P. Bidan, A. Rumeau, H. Morel, X. Perpina, M. Vellvehi, S. M'Rad, Reduced-Order Thermal Behavioral Model Based on Diffusive Representation, Power Electron. IEEE Trans. 24 (12) (2009) 2833-2846. doi:10.1109/TPEL.2009.2028231

580 [13] M. Dominguez-Pumar, M. T. Atienza, L. Kowalski, S. Novio, S. Gorreta, V. Jimenez, S. Silvestre, Heat flow dynamics in thermal systems described by diffusive representation, IEEE Transactions on Industrial Electronics PP (99) (2016) 1-1. doi:10.1109/TIE. 2016.2605621

585 [14] G. Montseny, Representation diffusive, Lavoisier, 2005.

[15] L. Laudebat, P. Bidan, G. Montseny, Modeling and optimal identification of pseudodifferential electrical dynamics by means of diffusive representationpart i: modeling, Circuits and Systems I: Regular Papers, IEEE Trans. 51 (9) (2004) 1801-1813. doi:10.1109/TCSI . 2004.834501

[16] V. A. T. L. D. George I Bourdopoulos Aristodemos Pnevmatikakis, DeltaSigma Modulators: Modeling, Design and Applications, Imperial College Press, 2003.

[17] H. Sira-Ramírez, Sliding Mode Control. The Delta-Sigma Modulation Approach, Birkhäuser Basel, 2015.

[18] J. Davidson, D. Stone, M. Foster, D. Gladwin, Improved Bandwidth and Noise Resilience in Thermal Impedance Spectroscopy by Mixing PRBS Signals, Power Electron. IEEE Trans. 29 (9) (2014) 4817-4828. doi: 10.1109/TPEL.2013.2288936 